\title{
Somatic Complaints Are Significantly Associated with Chronic Uninvestigated Dyspepsia and Its Symptoms: A Large Cross-sectional Population Based Study
}

\author{
Zahra Heidari, ${ }^{1}$ Ammar Hassanzadeh Keshteli, ${ }_{1}^{2,3}$ Awat Feizi, ${ }^{1,4 *}$ Hamid Afshar, ${ }^{4}$ and Payman Adibi ${ }^{3,5}$ \\ ${ }^{1}$ Department of Biostatistics and Epidemiology, School of Health, Isfahan University of Medical Sciences, Isfahan, Iran; ${ }^{2}$ Department of Medicine, \\ University of Alberta, Edmonton, Alberta, Canada; ${ }^{3}$ Integrative Functional Gastroenterology Research Center, Isfahan University of Medical \\ Sciences, Isfahan, Iran; ${ }^{4}$ Psychosomatic Research Center, Isfahan University of Medical Sciences, Isfahan, Iran; and ${ }^{5}$ Division of Gastroenterology \\ and Hepatology, Department of Internal Medicine, School of Medicine Isfahan University of Medical Sciences, Isfahan, Iran
}

\begin{abstract}
Background/Aims
Somatization may influence persistence and severity of symptoms in gastrointestinal diseases. Some studies suggest that somatization is associated with chronic uninvestigated dyspepsia (CUD); however, the association is unclear. We aimed to determine the association between the profiles of somatic complaints with CUD and its symptoms.

\section{Methods}

In a cross-sectional study conducted on 4763 Iranian adults, somatic complaints were assessed using a comprehensive 31-items questionnaire. Patients with CUD were identified by the Rome III diagnostic criteria. Profiles of somatic complaints were derived from factor analysis. Logistic regression analysis was used to assess the relationship between extracted profiles with CUD and its symptoms.

\section{Results}

CUD, bothersome postprandial fullness, early satiation, and epigastric pain or burning was identified in $723(15.2 \%), 384(8.1 \%)$, $302(6.3 \%)$, and $371(7.8 \%)$ of the study population. The frequency of all 31 somatic complaints was significantly higher in patients with CUD compared with controls $(P<0.001)$, and the most frequent was severe fatigue $(45.1 \%)$. The profiles of somatic complaints were extracted in 4 domains, including "psychological", "gastrointestinal", "neuro-skeletal", and "pharyngeal-respiratory". The psychological (OR, 1.49; 95\% Cl, 1.44-1.54), gastrointestinal (OR, 2.22; 95\% Cl, 2.09-2.37), neuro-skeletal $(\mathrm{OR}, 1.52 ; 95 \% \mathrm{Cl}, 1.44$ 1.59), and pharyngeal-respiratory (OR, 2.09; $95 \% \mathrm{Cl}, 1.94-2.25)$ profiles were significantly associated with greater odds of CUD.
\end{abstract}

\section{Conclusions}

CUD and its symptoms are strongly associated with higher levels of somatic complaints and their related extracted profiles. This perhaps explains that why it can be difficult to treat, however further prospective investigations are required to confirm these associations.

(J Neurogastroenterol Motil 2017;23:80-91)

\section{Key Words}

Dyspepsia; Gastrointestinal diseases; Mental disorders; Psychosomatic disorders

Received: February 4, 2016 Revised: June 10, 2016 Accepted: July 4, 2016

(.) This is an Open Access article distributed under the terms of the Creative Commons Attribution Non-Commercial License (http://creativecommons. org/licenses/by-nc/4.0) which permits unrestricted non-commercial use, distribution, and reproduction in any medium, provided the original work is properly cited.

*Correspondence: Awat Feizi, PhD Department of Biostatistics and Epidemiology, School of Public Health and Psychosomatic Research Center, Isfahan University of Medical Sciences, Hezarjarib St, Isfahan 81745, Iran Tel: +98-3137923250, Fax: +98-3137923253,E-mail: awat_feiz@hlth.mui.ac.ir 


\section{Introduction}

Functional dyspepsia (FD) is one of the highly prevalent gastrointestinal (GI) disorders which is defined by the presence of symptoms originating from the gastroduodenal region, without identifiable cause by conventional diagnostic means. ${ }^{1-4}$ Under the Rome III criteria, the FD symptoms include postprandial fullness, early satiation, epigastric pain or burning. ${ }^{5}$ In addition, other symptoms may coexist, such as bloating (may be derived from the bowel), nausea (often of central origin), vomiting, belching, and heartburn (esophageal origin). ${ }^{4,6,7}$ The dyspeptic symptoms reduce substantially the quality of life and greatly increase health costs. ${ }^{8-12}$ Epidemiological studies reported a considerably high range of FD prevalence over the world. The prevalence of FD has been estimated to be $20-30 \%$ in the general population, ${ }^{13} 8-23 \%$ and 20 $30 \%$ in Asian and Western populations, respectively ${ }^{2,5,14,15}$ and $8.9 \%$ in Iran. ${ }^{16}$

The etiology of FD is incompletely understood and it is believed to be multi-factorial. ${ }^{17,18}$ Reported factors include gastric sensorimotor dysfunction, Helicobacter pylori infection, dysmotility, psychosocial factors (anxiety, anger-hostility, depression, and general psychological distress), and personality traits (among them, neuroticism). ${ }^{17-23}$ In addition, somatization, which is characterized as physical bodily complaints not fully explained by organic reasons, plays an important role in functional GI disorders in general and in FD in particular. ${ }^{23-26}$ Some studies indicated that there is a considerable overlap of somatic complaints like fatigue, headaches, sleep disorders, and abdominal discomfort in patients with FD, and that these are associated with epigastric pain, epigastric and retrosternal burning, and total dyspeptic symptom scores. ${ }^{25,27,28}$ Despite several studies that reported high levels of somatization among patients with $\mathrm{FD},{ }^{18,23,28-30}$ few have examined the frequency of somatization varies between FD patients and healthy counterparts. ${ }^{18}$ In addition, to our knowledge, there is no data concerning the association of specific somatic complaint profiles with FD and its symptoms.

This highlights the need for a comprehensive study on examining the relationship between chronic uninvestigated dyspepsia (CUD) and its symptoms with somatic complaints. We have therefore examined this issue in a large group of patients with Rome IIIdefined CUD, and a control group of participants without CUD. We postulated that the frequency of somatic complaints would be greater in those with CUD than controls, and higher levels of somatic complaints profiles would be associated with greater odds of CUD and its symptoms.

\section{Materials and Methods}

\section{Study Design and Participants}

This cross-sectional study was conducted in the framework of the Study on the Epidemiology of Psychological, Alimentary Health and Nutrition (SEPAHAN) project that was performed in 2 phases in a large sample of Iranian population adults in the Isfahan province. ${ }^{31}$ In the first phase of SEPAHAN, which included different questionnaires on demographic information, lifestyle, and nutritional factors, among 10087 persons invited to participate, 8691 subjects took part (response rate: $86.16 \%$ ). After this phase, the second wave of questionnaires which was designed to gather information on GI and psychological profiles, somatic complaints and personality traits questionnaires of participants were distributed, and 6239 completed questionnaires were returned to the main office of the project (response rate: $64.64 \%$ ). Then, national identification numbers of the subjects were used to link the questionnaires from both waves. We collected and matched the information from both phases and after considering missing data, data on 4763 subjects with completed information was used in the current analysis. Fourteen questionnaires were used in the SEPAHAN project, among them, according to the objective of the current study, 8 questionnaires were considered. Informed consent was obtained from all subjects. The Bioethics Committee of the Isfahan University of Medical Sciences, Isfahan, Iran, approved the study (IUMS) (Project No. 189069, 189082, and 189086).

\section{Procedures and Assessment of Variables}

\section{Assessment of chronic uninvestigated dyspepsia}

In this study, a modified Persian version of the Rome III questionnaire was used for the assessment of functional GI disorders, including CUD. Face validation of this questionnaire indicated that most participants could not discriminate the difference between the descriptors (the rating scales) used in the original Rome III questionnaire (never, less than one day a month, one day a month, 2 to 3 days a month, one day a week, more than one day a week, and every day). Therefore, rating scales were modified to 4 descriptors (ie, never or rarely, sometimes, often, always) for each question. ${ }^{32}$ In the current study, CUD was recognized having one or more of the following characteristics: bothersome postprandial fullness (defined as feeling uncomfortably full after a regular-sized meal, often or always), early satiation (defined as being unable to finish 
a regular-sized meal, often or always), and/or epigastric pain or epigastric burning (defined as feeling pain or burning in the middle of abdomen, often or always). The validity of the Persian version of the Rome III questionnaire in an Iranian population has been determined previously. ${ }^{16}$ Controls were all other participants who did not meet the Rome III criteria for CUD.

\section{Assessment of somatization}

In the SEPAHAN project, there is not separate questionnaire to assess somatic complaints, however, we found 31 common items among questions contained in SEPAHAN's questionnaires with the 47-items questionnaire used in the Lacourt et al's study ${ }^{33}$ and the patient health questionnaire (PHQ), ${ }^{34-37}$ as standard tools for the assessment of somatic complaints, and therefore we established a 31-item validated questionnaire. This was used to evaluate the frequency of somatic complaints. Respondents could indicate how much they had experienced each symptom in the past three months on a 4-point Likert scale ( 1 = never, sometimes, often, always). For one item (ie, dry mouth), the rating scale was as: never, low and high. We conducted a separate mini survey of 100 participants selected randomly in order to assess the reliability of this instrument. There was strong internal reliability, with a Cronbach's alpha score of 0.903 .

\section{Assessment of other variables}

Self-administered standard questionnaires were distributed to collect information on age (years), gender (male/female), marital status (married, single), self-reported weight $(\mathrm{kg})$, and height $(\mathrm{cm})$. Body mass index (BMI) was calculated by dividing the weight in kilograms by height in meters squared. Educational attainments categorized into 3 categories as lower than diploma (12 years' formal education), diploma, and higher than diploma (including bachelor, master, and doctorate). An Iranian validated self-administered, Hospital Anxiety and Depression Scale (HADS) questionnaire was used to assess anxiety and depression. ${ }^{38}$ It consisted of 14 items of which 7 are allocated to anxiety. It was answered using a 4-point Likert scale ranging from 0 (not present) to 3 (considerable). The anxiety score of participants could be between 0 and 21 points (0-7 normal, 8-21 mild, moderate or severe disorder). Psychological distress was measured by an Iranian validated self-administered questionnaire ie, a 12-item general health questionnaire (GHQ12). ${ }^{39}$ A participant's score could be between 0 and 12 points, and a threshold score of 4 or more was used to identify a participant with high-distress level. ${ }^{40}$ The stressful life event (SLE) questionnaire was used to determine the intensity of perceived stress of subjects.
SLE consisted eleven domains, including home life, financial problems, social relations, personal conflicts, job conflicts, educational concerns, job security, loss and separation, sexual life, daily life, and health concerns. The validity of the questionnaire has been evaluated in a large sample of Iranian adults with Cronbach's $\alpha=92 \%$ and acceptable correlation among SLE domains and SLE with GHQ-12. ${ }^{41}$ The Neuroticism-Extraversion-Openness Five-Factor Inventory (NEO-FFI) was used to determine personality traits of subjects. The NEO-FFI results in a profile of the personality of the subject and consisted of 60 self-descriptive statements (a 5-point Likert-type scale including $0=$ strongly disagree, $4=$ strongly agree) about the personality that measured five dimensions of the normal personality (ie, neuroticism, extraversion, openness, agreeableness, and conscientiousness). ${ }^{42}$ General practice physical activity questionnaire (GPPAQ) was used to assess an individual's current physical activity status. The GPPAQ consists of work and leisure time physical activity. It generates a simple, 4-levels physical activity index categorizing subjects as: active, moderately active, moderately inactive, and inactive. ${ }^{43}$ In the current analysis, participants were classified into 2 categories ie, inactive (including inactive and moderately inactive) and active (including moderately active and active) as indicated earlier.

\section{Statistical Methods}

Continuous and categorical variables were represented as mean (SE) and frequency (percentage), respectively. To compare the continuous variables between patients with CUD and controls, Independent Student's $t$ test was conducted. Distribution of study participants in terms of categorical variables and the number with the levels of "often and always" of the 31 individual somatic complaints was compared between those with CUD and controls using the Chi-square test.

Profiles of somatic complaints were extracted using factor analysis based on the principal component extraction approach on the 31 individual somatic complaints. The orthogonal varimax rotation procedure was used to find the interpretable factors. Factors were retained for further analysis based on their natural interpretation and eigenvalues on the scree plot. In this study, we retained factors with eigenvalues $>2$ as this cutoff could result in more interpretable somatic complaints profiles and explain sufficient amounts of overall variation. Four main somatic complaints profiles were labeled based on the loaded somatic complaints in each factor. The factor score for each profile was computed by summing up items of somatic complaints weighted by their factor loadings and assigned to each participant. 
To assess the relationship between extracted factors of somatic complaints and CUD, logistic regression analysis was performed in different models. Also, to assess the association between profiles of somatic complaints, as the main independent variables, and fre- quency (never, sometimes, often, and always) of symptoms of CUD (bothersome postprandial fullness, early satiation, and epigastric pain or burning) as the outcome variables, we applied proportional odds ordinal logistic regression. Adjusted ORs with 95\% CI for

Table 1. Demographic, Life Style, and Psychological Characteristics of Study Participants

\begin{tabular}{|c|c|c|c|}
\hline Characteristics & CUD $(n=723)$ & Control $(n=4040)$ & $P$-value ${ }^{a}$ \\
\hline \multicolumn{4}{|l|}{ Demographic characteristics } \\
\hline Age (yr) & $36.17 \pm 0.32$ & $36.65 \pm 0.14$ & 0.174 \\
\hline Females & $457(63.2)$ & $2200(54.5)$ & $<0.001$ \\
\hline Marital status & & & 0.748 \\
\hline Married & $578(81.6)$ & $3198(81.1)$ & \\
\hline Single & $130(18.4)$ & $744(18.9)$ & \\
\hline Educational Level & & & $<0.001$ \\
\hline Under Diploma (< 12 yr) & $132(18.8)$ & $506(12.9)$ & \\
\hline Diploma (12 years) & $233(33.1)$ & $1115(28.3)$ & \\
\hline University Graduate (> 12 yr) & $338(48.1)$ & $2312(58.8)$ & \\
\hline \multicolumn{4}{|l|}{ Life style } \\
\hline BMI & $24.61 \pm 0.18$ & $25.04 \pm 0.06$ & 0.022 \\
\hline Physical activity & & & $<0.001$ \\
\hline Inactive and moderately inactive & $496(72.4)$ & $2359(63.9)$ & \\
\hline Moderately active and active & $189(27.6)$ & $1333(36.1)$ & \\
\hline \multicolumn{4}{|l|}{ Psychological characteristics } \\
\hline Psychological distress & & & $<0.001$ \\
\hline No & $404(57.1)$ & $3157(80.5)$ & \\
\hline Yes & $304(42.9)$ & $763(19.5)$ & \\
\hline Anxiety & & & $<0.001$ \\
\hline No & $458(64.5)$ & $3545(89.8)$ & \\
\hline Borderline & $128(18.0)$ & $257(6.5)$ & \\
\hline Yes & $124(17.5)$ & $145(3.7)$ & \\
\hline Depression & & & $<0.001$ \\
\hline No & $327(46.0)$ & $2988(75.8)$ & \\
\hline Borderline & $202(28.4)$ & $653(16.6)$ & \\
\hline Yes & $182(25.6)$ & $301(7.6)$ & \\
\hline Intensity of perceived stress & & & $<0.001$ \\
\hline No at risk & $110(15.2)$ & $1315(32.5)$ & \\
\hline At risk & $613(84.8)$ & $2725(67.5)$ & \\
\hline \multicolumn{4}{|l|}{ Personality traits } \\
\hline Neuroticism score & $22.69 \pm 0.29$ & $18.02 \pm 0.12$ & $<0.001$ \\
\hline Extraversion score & $26.68 \pm 0.26$ & $29.45 \pm 0.11$ & $<0.001$ \\
\hline Openness score & $23.69 \pm 0.20$ & $24.11 \pm 0.08$ & 0.047 \\
\hline Agreeableness score & $29.50 \pm 0.24$ & $31.33 \pm 0.10$ & $<0.001$ \\
\hline Conscientiousness score & $34.69 \pm 0.27$ & $36.55 \pm 0.11$ & $<0.001$ \\
\hline The chronic and acute diseases ${ }^{\mathrm{b}}$ & & & $<0.001$ \\
\hline No & $532(73.6)$ & $3453(85.5)$ & \\
\hline Yes & $191(26.4)$ & $587(14.5)$ & \\
\hline
\end{tabular}

${ }^{a} P$-values from independent samples $t$ test for continuous data and from Pearson $\chi^{2}$ for categorical data.

${ }^{b}$ Experiencing at least one of the chronic and acute diseases including high blood cholesterol and triglycerides (lipid disorder), high blood pressure (hypertension), diabetes mellitus, asthma, gallstones, Crohn's disease, stroke, heart attack, heart failure, and cancer.

CUD, chronic uninvestigated dyspepsia; BMI, body mass index.

Values are mean $\pm \mathrm{SE}$ and others are number $(\%)$. 
CUD and its symptoms are presented in 4 different models. First, we adjusted for demographic variables including age, gender, marital status, and educational level. In the second model, further adjustments were made for lifestyle variables including BMI and physical activity. Additional adjustment was conducted for psychological disorders (anxiety, depression, and intensity of perceived stress) in the third model. Personality traits, including neuroticism, extraversion, openness, agreeableness, and conscientiousness were adjusted in the fourth model. During the fitting of ordinal logistic regression, the proportional odd assumption or parallel lines test was checked using the Chi-square test. Data analyses were performed using Statistical Package for Social Sciences version 15 (IBM Corp, Armonk, NY, USA). $P$-values less than 0.05 were considered statistically significant.

\section{Results}

CUD was found in 723 (15.2\%) of the study samples. Symptoms of bothersome postprandial fullness, early satiation, and epigastric pain or burning were identified in 384 (8.1\%), 302 (6.3\%),

Table 2. Prevalence of Individual Somatic Complaints in Chronic Uninvestigated Dyspepsia Patients Compared with Controls

\begin{tabular}{|c|c|c|c|c|}
\hline \multirow{2}{*}{ Somatic Complaints } & \multicolumn{2}{|c|}{ CUD } & \multicolumn{2}{|c|}{ Controls } \\
\hline & Often & Always & Often & Always \\
\hline Sleep disorder & $186(26.8)$ & $63(9.1)$ & $345(9.0)$ & $69(1.8)$ \\
\hline Pounding heart & $128(18.1)$ & $34(4.8)$ & $156(3.9)$ & $33(0.8)$ \\
\hline Feeling low on energy & $197(27.7)$ & $117(16.5)$ & $498(12.7)$ & $181(4.6)$ \\
\hline Feeling like "butterflies" in the stomach & $128(18.2)$ & $63(9.0)$ & $140(3.6)$ & $55(1.4)$ \\
\hline Difficulty concentrating & $128(18.2)$ & $63(9.0)$ & $140(3.6)$ & $55(1.4)$ \\
\hline Disturbing thoughts & $154(21.8)$ & $98(13.9)$ & $362(9.2)$ & $168(4.3)$ \\
\hline Dry mouth $^{\mathrm{a}}$ & $284(40.1)$ & $70(9.9)$ & $1113(28.2)$ & $99(2.5)$ \\
\hline Chest pain & $133(18.8)$ & $19(2.7)$ & $90(2.3)$ & $10(0.3)$ \\
\hline Feeling of fullness & $320(44.9)$ & $64(9.0)$ & $0(0.0)$ & $0(0.0)$ \\
\hline Nausea & $74(10.6)$ & $29(4.2)$ & $44(1.1)$ & $9(0.2)$ \\
\hline Gastroesophageal reflux & $53(7.7)$ & $21(3.1)$ & $27(0.7)$ & $6(0.2)$ \\
\hline Pain or discomfort in the abdomen & $206(29.4)$ & $49(7.0)$ & $152(3.9)$ & $17(0.4)$ \\
\hline Constipation & $143(20.3)$ & $62(8.8)$ & $272(6.9)$ & $44(1.1)$ \\
\hline Diarrhea & $31(4.4)$ & $5(0.7)$ & $38(1.0)$ & $5(0.1)$ \\
\hline Bloating or swelling of the abdomen & $209(30.3)$ & $101(14.7)$ & $280(7.3)$ & $66(1.7)$ \\
\hline Anal pain & $49(7.3)$ & $13(1.9)$ & $62(1.6)$ & $7(0.2)$ \\
\hline Headache & $224(31.5)$ & $67(9.4)$ & $502(12.8)$ & $84(2.1)$ \\
\hline Back pain & $182(25.7)$ & $86(12.2)$ & $466(11.9)$ & $154(3.9)$ \\
\hline Pain in joints & $170(24.0)$ & $100(14.1)$ & $341(8.6)$ & $139(3.5)$ \\
\hline Eyesore & $107(15.2)$ & $37(5.3)$ & $202(5.1)$ & $40(1.0)$ \\
\hline Severe fatigue & $320(45.1)$ & $118(16.6)$ & $810(20.6)$ & $164(4.2)$ \\
\hline Dizziness and confusion & $123(17.3)$ & $40(5.6)$ & $171(4.3)$ & $23(0.6)$ \\
\hline Chills and extreme cold & $58(8.2)$ & $10(1.4)$ & $63(1.6)$ & $12(0.3)$ \\
\hline Hot flashes & $95(13.4)$ & $25(3.5)$ & $132(3.3)$ & $25(0.6)$ \\
\hline Menstrual disorder ${ }^{\mathrm{b}}$ & $94(21.8)$ & $45(10.4)$ & $238(11.3)$ & $132(6.3)$ \\
\hline Neck pain & $75(10.6)$ & $13(1.8)$ & $88(2.2)$ & $17(0.4)$ \\
\hline Globus sensation & $63(8.9)$ & $17(2.4)$ & $50(1.3)$ & $11(0.3)$ \\
\hline Having trouble swallowing & $31(4.3)$ & $3(0.4)$ & $20(0.5)$ & $2(0.1)$ \\
\hline Shortness of breath & $96(13.6)$ & $41(5.8)$ & $111(2.8)$ & $30(0.8)$ \\
\hline Hoarseness & $40(5.7)$ & $5(0.7)$ & $33(0.8)$ & $11(0.3)$ \\
\hline Wheezing (asthma) & $28(4.0)$ & $14(2.0)$ & $49(1.2)$ & $9(0.2)$ \\
\hline
\end{tabular}

${ }^{a}$ The rating scale was as "never, low, and high."

${ }^{\mathrm{b}}$ Female patients only.

Values are number $(\%)$

$P$-value from Pearson $\chi^{2}$. Distribution of all studied variables is significantly different between 2 groups at $P<0.001$. 
and $371(7.8 \%)$ of subjects, respectively. The mean age was 36.17 \pm 0.32 years in patients with CUD. Distribution of demographic, life style, psychological characteristics, and personality traits of study participants are summarized in Table 1. Patients with CUD were female predominant (63.2\%), and they had lower levels of physical activities than controls $(P<0.001)$. Also, patients with CUD had higher anxiety, depression, psychological distress, intensity of perceived stress, and neuroticism scores $(P<0.001)$ while they had lower BMI, extraversion, openness, agreeableness, and conscientiousness scores $(P<0.05)$. The prevalence of experiencing at least one of the chronic and acute diseases including "high blood cholesterol and triglycerides (lipid disorder), high blood pressure (hypertension), diabetes mellitus, asthma, gallstones, Crohn's disease, stroke, heart attack, heart failure, and cancer" was significantly higher in patients with CUD $(26.4 \%)$ compared with controls $(14.5 \%)(P<0.001)$ (Table 1$)$

\section{Prevalence of Somatic Complaints Among Chronic Uninvestigated Dyspepsia Patients and Controls}

The prevalence of the somatic complaints among patients with CUD and controls is presented in Table 2. The majority of symptoms had a 4-point Likert scale; we only reported the response of

Table 3. Factor Loadings for the 4 Extracted Somatic Complaints Profiles from 31 Somatic Complaints

\begin{tabular}{|c|c|c|c|c|}
\hline \multirow{2}{*}{ Somatic complaints } & \multicolumn{4}{|c|}{ Factor loadings $^{\mathrm{a}}$} \\
\hline & Psychological & Gastrointestinal & Neuro-skeletal & Pharyngeal-respiratory \\
\hline Sleep disorder & 0.420 & & & \\
\hline Pounding heart & 0.577 & & & \\
\hline Feeling low on energy & 0.565 & & & \\
\hline Feeling like "butterflies" in the stomach & 0.869 & & & \\
\hline Difficulty concentrating & 0.869 & & & \\
\hline Disturbing thoughts & 0.664 & & & \\
\hline Dry mouth & & 0.248 & & \\
\hline Chest pain & & 0.454 & & \\
\hline Feeling of fullness & & 0.662 & & \\
\hline Nausea & & 0.509 & & \\
\hline Gastroesophageal reflux & & 0.544 & & \\
\hline Pain or discomfort in the abdomen & & 0.678 & & \\
\hline Constipation & & 0.512 & & \\
\hline Diarrhea & & 0.374 & & \\
\hline Bloating or swelling of the abdomen & & 0.644 & & \\
\hline Anal pain & & 0.473 & & \\
\hline Headache & & & 0.547 & \\
\hline Back pain & & & 0.661 & \\
\hline Pain in joints & & & 0.612 & \\
\hline Eyesore & & & 0.461 & \\
\hline Severe fatigue & & & 0.646 & \\
\hline Dizziness and confusion & & & 0.513 & \\
\hline Chills and extreme cold & & & 0.435 & \\
\hline Hot flashes & & & 0.350 & \\
\hline Menstrual disorder & & & 0.389 & \\
\hline Neck pain & & & & 0.570 \\
\hline Globus sensation & & & & 0.510 \\
\hline Having trouble swallowing & & & & 0.596 \\
\hline Shortness of breath & & & & 0.492 \\
\hline Hoarseness & & & & 0.612 \\
\hline Wheezing (asthma) & & & & 0.567 \\
\hline Variance explained (\%) & 12.70 & 11.73 & 11.55 & 8.85 \\
\hline Cumulative variance & 12.70 & 24.43 & 35.99 & 44.84 \\
\hline
\end{tabular}

${ }^{\mathrm{a}}$ Factor loadings $<0.2$ are not shown for simplicity. 
participants to "often and always" categories. patients with CUD reported all 31 somatic complaints at both levels significantly higher than controls $(P<0.001)$. In the "often" level, the commonest somatic complaints reported among patients with CUD were severe fatigue (45.1\%), followed by feelings of fullness (44.9\%) and headaches (31.5\%). In the "always" category, the most frequent somatic complaints reported among patients with CUD were severe fatigue (16.6\%), followed by feeling low on energy (16.5\%), bloating or swelling of the abdomen (14.7\%), pain in joints (14.1\%) disturbing thoughts (13.9\%), back pains (12.2\%), and menstrual disorders (10.4\%) (Table 2).

\section{Extraction of Somatic Complaints Profiles Using Factor Analysis}

Profiles of somatic complaints were extracted using factor analysis based on principal component extraction approach on the 31 individual somatic complaints. Thus, four factors were extracted and labeled based on value of loadings within each factor as "psychological," "gastrointestinal," "neuro-skeletal," and "pharyngealrespiratory" (Table 3). The 4 factors accounted for $12.70 \%$, $11.73 \%, 11.55 \%$, and $8.85 \%$, respectively, of the total variance.

Table 4. Crude and Adjusted Odds Ratio (95\% Confidence Interval) for Association Between Somatic Complaints Profiles with Chronic Uninvestigated Dyspepsia and Its Symptoms

\begin{tabular}{|c|c|c|c|c|}
\hline \multirow{2}{*}{ Dependent variables } & \multicolumn{4}{|c|}{ Somatic complaints profiles } \\
\hline & Psychological & Gastrointestinal & Neuro-skeletal & Pharyngeal-respiratory \\
\hline \multicolumn{5}{|l|}{ Crude Model } \\
\hline CUD & $1.49(1.44-1.54)$ & $2.22(2.09-2.37)$ & $1.52(1.44-1.59)$ & $2.09(1.94-2.25)$ \\
\hline Bothersome postprandial fullness & $1.47(1.43-1.51)$ & $3.00(2.84-3.18)$ & $1.52(1.46-1.58)$ & $2.12(1.99-2.25)$ \\
\hline Early satiation & $1.36(1.32-1.40)$ & $1.65(1.59-1.71)$ & $1.42(1.36-1.47)$ & $1.90(1.79-2.02)$ \\
\hline Epigastric pain or burning & $1.45(1.41-1.50)$ & $2.20(2.10-2.30)$ & $1.50(1.44-1.59)$ & $2.05(1.93-2.17)$ \\
\hline \multicolumn{5}{|l|}{ Model I } \\
\hline CUD & $1.47(1.41-1.53)$ & $2.23(2.08-2.39)$ & $1.50(1.41-1.58)$ & $2.01(1.85-2.18)$ \\
\hline Bothersome postprandial fullness & $1.46(1.42-1.51)$ & $3.03(2.84-3.22)$ & $1.51(1.44-1.57)$ & $2.09(1.95-2.23)$ \\
\hline Early satiation & $1.36(1.32-1.40)$ & $1.67(1.60-1.74)$ & $1.44(1.37-1.50)$ & $1.91(1.78-2.04)$ \\
\hline Epigastric pain or burning & $1.44(1.40-1.49)$ & $2.16(2.06-2.27)$ & $1.48(1.42-1.55)$ & $2.04(1.91-2.18)$ \\
\hline \multicolumn{5}{|l|}{ Model II } \\
\hline CUD & $1.46(1.40-1.52)$ & $2.24(2.08-2.41)$ & $1.50(1.41-1.60)$ & $2.02(1.85-2.21)$ \\
\hline Bothersome postprandial fullness & $1.46(1.41-1.51)$ & $3.09(2.89-3.31)$ & $1.51(1.44-1.58)$ & $2.12(1.97-2.28)$ \\
\hline Early satiation & $1.38(1.33-1.42)$ & $1.73(1.65-1.81)$ & $1.49(1.42-1.57)$ & $2.02(1.87-2.17)$ \\
\hline Epigastric pain or burning & $1.43(1.38-1.48)$ & $2.16(2.05-2.27)$ & $1.50(1.43-1.60)$ & $2.08(1.94-2.24)$ \\
\hline \multicolumn{5}{|l|}{ Model III } \\
\hline CUD & $1.56(1.43-1.70)$ & $2.20(2.03-2.38)$ & $1.36(1.26-1.46)$ & $1.69(1.54-1.86)$ \\
\hline Bothersome postprandial fullness & $1.51(1.41-1.61)$ & $3.10(2.88-3.34)$ & $1.37(1.30-1.45)$ & $1.75(1.62-1.89)$ \\
\hline Early satiation & $1.31(1.23-1.41)$ & $1.63(1.55-1.72)$ & $1.35(1.27-1.43)$ & $1.70(1.56-1.84)$ \\
\hline Epigastric pain or burning & $1.56(1.45-1.66)$ & $2.13(2.01-2.25)$ & $1.38(1.30-1.46)$ & $1.75(1.62-1.89)$ \\
\hline \multicolumn{5}{|l|}{ Model IV } \\
\hline CUD & $1.57(1.44-1.71)$ & $2.21(2.04-2.40)$ & $1.36(1.26-1.46)$ & $1.69(1.53-1.86)$ \\
\hline Bothersome postprandial fullness & $1.52(1.42-1.63)$ & $3.12(2.90-3.36)$ & $1.38(1.30-1.46)$ & $1.75(1.62-1.89)$ \\
\hline Early satiation & $1.32(1.23-1.42)$ & $1.64(1.55-1.73)$ & $1.36(1.28-1.44)$ & $1.69(1.56-1.84)$ \\
\hline Epigastric pain or burning & $1.56(1.46-1.67)$ & $2.13(2.02-2.26)$ & $1.38(1.30-1.46)$ & $1.75(1.62-1.90)$ \\
\hline
\end{tabular}

CUD, chronic uninvestigated dyspepsia.

Model I: adjusted for age, gender, marital status, and educational level; Model II: adjusted for age, gender, marital status, educational level, body mass index (BMI), and physical activity; Model III: adjusted for age, gender, marital status, educational level, BMI, physical activity, and psychological disorders (anxiety, depression, and intensity of perceived stress); Model IV: adjusted for all variables in model III, personality traits (neuroticism, extraversion, openness, agreeableness, and conscientiousness) and chronic and acute diseases.

Values obtained from binary logistic regression for CUD and ordinal logistic regression for its symptoms. 


\section{Association Between Somatic Complaints Profiles with Chronic Uninvestigated Dyspepsia and Its Symptoms}

Crude and adjusted OR and 95\% CI for the association between somatic complaints profiles and CUD and its symptoms in the entire study population are presented in Table 4. Significant associations were found between all somatic complaint profiles with CUD and its symptoms in crude models and adjusted models. In the crude model and also after adjustment for demographic variables, BMI and physical activity, and the psychological profile was significantly associated with greater odds of CUD (OR, 1.46; 95\% CI, 1.40-1.52). This association remained significant even after further controlling for psychological disorders (anxiety, depression, intensity of perceived stress) and personality traits $(1.57 ; 1.44-1.71)$. Similar associations were found between the psychological profile and symptoms of CUD including bothersome postprandial fullness and epigastric pain or burning (nearly 1.5; 95\% CI, 1.4-1.6 for last model). The psychological profile had significant association with symptom of early satiation too $(1.32 ; 1.23-1.42)$. The GI profile was significantly associated with greater odds of CUD in the crude model and adjusted models $(2.2 ; 2.0-2.4)$. In the crude model and also after adjustment for demographic variables, BMI, physical activity, psychological disorders, and personality traits, the GI profile was significantly associated with greater odds of bothersome postprandial fullness $(3.12 ; 2.90-3.36)$. In all models, the GI profile was significantly associated with greater odds of early satiation (1.64; $1.55-1.73$ for the last model) and epigastric pain or burning $(2.1 ; 2.0-2.3)$. In the crude model and also after adjustment for demographic variables, BMI, and physical activity, the neuro-skeletal profile was significantly associated with greater odds of CUD (1.5; 1.4-1.6). This association remained significant even after further controlling for psychological disorders and personality traits (1.36; 1.26-1.46). Similar associations were found between the neuroskeletal profile and symptoms of CUD including bothersome postprandial fullness and epigastric pain or burning (nearly 1.38; 1.30-1.46 for last model). The neuro-skeletal profile had significant association with symptom of early satiation too $(1.36 ; 1.28-1.44)$. The pharyngeal-respiratory factor was significantly related to the increased risk of CUD in the crude model and also after adjustment for demographic variables, BMI, and physical activity (2.0; 1.9-2.2). This association remained significant even after further controlling for psychological disorders and personality traits (1.69; 1.53-1.86). Similar associations were found between the pharyngeal-respiratory profile with bothersome postprandial fullness and epigastric pain or burning $(1.75 ; 1.62-1.9)$. In the crude model, the pharyngealrespiratory factor was significantly associated with greater odds of early satiation $(1.90 ; 1.79-2.02)$. This association remained significant even after controlling for the effects of potential confounding variables $(1.69 ; 1.56-1.84)$.

\section{Discussion}

This cross-sectional study among a large sample of Iranian adults demonstrated that patients with CUD had a significantly higher prevalence of all somatic complaints than controls. When assessing the relationship between somatic complaint profiles extracted from factor analysis and CUD and its symptoms, we found that the psychological, neuro-skeletal, and pharyngeal-respiratory profiles were significantly associated with greater odds of CUD, bothersome postprandial fullness, early satiation, and epigastric pain or burning symptoms. Our study also reconfirmed an expecting relationship between the GI profile with greater odds of CUD and its symptoms. All aforesaid associations were observed with and without taking potential confounders into account.

The majority of previous studies were restricted to investigation of the association between psychological factors and FD which include a few somatic complaints or an overall score of somatization. $^{23,29}$ To the best of our knowledge, our research is the first large epidemiological study on the evaluation of the associations of distinct profiles of somatic complaints identified by factor analysis and CUD and its symptoms. Some studies indicated that in FD, somatization correlates with epigastric pain, epigastric and retrosternal burning, and total dyspeptic symptom scores. ${ }^{27,28}$ In the present study, not only the most important somatic complaints in groups with and without CUD were compared, but also the conducted factor analysis provided us the possibility for evaluating the effect of extracted common concepts ie, somatic profiles on CUD and its symptoms.

In the current study, the extracted psychological profile from somatic complaints was one of the most important determinants of CUD and its symptoms. Also, our study showed significantly higher anxiety, depression, psychological distress, intensity of perceived stress, and neuroticism score in patients with CUD, than controls. These findings are consistent with other previous studies. ${ }^{19-22,44}$ For example, Norton et $\mathrm{al}^{45}$ showed that participants suffering from functional dyspepsia showed significant differences in terms of anxiety and depression when compared with controls. The results of Filipović et $\mathrm{al}^{21}$ also approved these facts. Review studies such as that of Barry and Dinan also confirmed the co-morbidities of anxi- 
ety, depressive, somatoform disorders, and neuroticism in patients with functional dyspepsia. ${ }^{19}$ These psychological problems are commonly seen in most digestive disorders so that in the study of Van Oudenhove et al, ${ }^{46}$ anxiety, depression, and somatization were used as the most important variables on separating functional gastroduodenal disorders (FGD). The results of Faramarzi et $\mathrm{al}^{20}$ in Iran revealed that psychological problems (depression and anxiety) were more prevalent in FD patients. Nan et al, ${ }^{47}$ based on possible braingut interaction, indicated that psychological factors may not only be just considered as an effect of FD; but also they could be a result of dyspeptic symptoms. However, Vishnar et $\mathrm{al}^{48}$ obtained rather contradictory results; the results showed that, except for anxiety, no significant association was found between the other disorders and non-ulcer dyspepsia. Also De la Roca-Chiapas et al ${ }^{49}$ showed that depression was not outlined as a significant predictor of FD. High scores for neuroticism as well as an increased rate for somatization and social anxiety in FD patients was reported by Holtmann et al. ${ }^{50}$

We also found that the psychological profile was significantly associated with greater odds of bothersome postprandial fullness, early satiation, and epigastric pain. Among a few studies about the association between psychological and somatic complaints with symptoms of CUD, some have presented indications of independent associations between scores of dyspeptic symptoms, such as the postprandial distress syndrome, with overall psychopathological stress, and particularly, somatization and depression. ${ }^{30}$

The results of our study are not surprising: given the strong association between FD and psychiatric comorbidity, it seems likely that patients with more GI symptoms have also more severe psychologic symptoms. The best combination of dimensions for describing this psychosocial disturbance and its positive association with CUD are included: personality (anger reactivity-the tendency to react readily with anger — and neuroticism), chronic social stressor threat, poor emotional support, and increased age. This profile, which combines psychological, social, and biological elements, is prototypic of the most severe chronic stress scenario. This pathway could be considered a pathophysiological mechanism capable of explaining the difference in the severity of the psychological suffering. ${ }^{13,23}$

The current study showed a significantly higher prevalence of the GI somatic complaints among patients with CUD than controls, and positive associations were observed between CUD and its symptoms with GI profiles extracted from factor analysis approach. These findings are naturally expected because of the large overlap between gastroesophageal reflux disease (GERD), irritable bowel syndrome (IBS), and FD, and often they coexist in the same patient. There is great overlap between FD and GERD- like symptoms ${ }^{51}$; and this overlap is greatest between nonerosive and functional heartburn and FD in which the clinical spectrum that extends from FD to nonerosive reflux disease may be present in up to $70 \%$ of GERD disease in the community. ${ }^{52}$ This association might indicate a common pathophysiologic mechanism. ${ }^{53}$ The high overlaps between FD and its symptoms with other upper and lower GI disorders, along with their strength interactions with psychopathological factors notably anxiety, depression, psychological distress and psychological somatization profile, can be viewed as the most important implication in this regard. ${ }^{13,54}$

We observed in the neuro-skeletal domain of psychosomatic symptoms that severe fatigue is the commonest reported somatic complaint among patients with CUD. Some previous studies showed that FD is one of the most prevalent functional gastrointestinal disorder (FGID) with a high comorbidity with other FGIDs such as IBS, as well as with non-GI functional somatic syndromes such as chronic fatigue syndrome. Approximately $40 \%$ of FD patients have comorbid chronic fatigue-like symptoms; (non-GI) somatic symptom level is associated with both FD and chronic fatigue comorbidity. ${ }^{23}$

In the study of Bennett et $\mathrm{al}^{29}$ a few items such as fatigue, unpleasant taste, dry skin or eczema, and binge eating habits were considered as somatic complaints, and were shown as predictive of the combination of FD and IBS in general. ${ }^{29}$ In line with our study, in the aforesaid study, fatigue was most likely to be present in FD patients. ${ }^{29}$ Gracie et $\mathrm{al}^{18}$ showed that the commonest item reported among patients with FD was feeling tired or low in energy, followed by trouble sleeping, and back pain. Van Oudenhove et $\mathrm{al}^{23}$ showed that dyspepsia symptom severity is associated with depression, fatigue, and somatization. ${ }^{23}$ However, it should be noted that in the aforesaid study the somatization scores with no separate effects of each items or domains, were used; therefore it can only be considered as indicative of functional somatization. In our study, in line with Jiang et al's study, ${ }^{55}$ the prevalence of sleeping disorders, as a somatic complaint, was significantly higher in patients with FD than healthy controls. ${ }^{55}$ These findings strongly suggest that FD patients may be characterized by a generalized tendency to experience and report multiple somatic complaints. An important issue that remains to be elucidated is whether this tendency (often described as "somatization") needs to be conceptualized as a primary phenomenon ("functional somatization"), or rather as the result of a complex psychobiological process in which different psychological dimensions such as personality traits including neuroticism, anxiety, depression or negative affects lead to amplification of bodily signals, most likely through sensitization of pain-processing neural systems 
(secondary phenomenon, "presenting somatization"). ${ }^{23,56-58}$

Our study identified a somatic complaint profile that we named as the "pharyngeal-respiratory" factor. This somatic profile similar to other identified somatic complaints was positively associated with CUD and its symptoms. Also, the individual pharyngeal-respiratory symptoms assessed in our study were more prevalent in patients with CUD than control participants. These findings are strongly in line with the results of previous studies that have indicated significant association between upper GI disorders and respiratory symptoms and complications, in which high prevalence of respiratory disorders or symptoms such as asthma, chronic cough or hoarseness, wheezing, and shortness of breath have been indicated in these patients. These symptoms may be present singly or collectively and usually are assumed to be caused by irritation of the larynx and hypopharynx by gastric contents. ${ }^{59,60}$

It is important to recognize some strengths, as well as potential limitations of the present study. A major strength of our large sample epidemiological population based study is the application of the factor analysis technique to distinct profiles of somatic complaints, instead of considering them as a single complaint in their relationships with CUD and its symptoms. Furthermore, the mediating impact of various potential confounding variables such psychological problems, personality traits, physical activity, and demographic variables, in order to evaluate reliable associations between the main study variables, have been considered. Several limitations need to be considered when interpreting our findings. The limitation of the majority of questionnaires on evaluating somatic complaints as a self-report measure is that they cannot distinguish between "medically explained" and "unexplained" symptoms, ${ }^{61}$ which is an important feature of the somatization concept. ${ }^{24,62}$ Accordingly, it should be emphasized that the somatic complaints in our study can only be characterized as indicative of somatization. In this study, the investigated associations are restricted to CUD, not organic dyspepsia: accordingly, the observed results are generalized only to CUD. Due to the cross-sectional nature of the SEPAHAN design, we cannot infer cause-effect relationships from our findings; thus, to identify the real association between CUD and its symptoms and somatic complaints, further prospective cohort investigations are suggested. It should also be kept in mind, as a common problem of epidemiological studies, that all used information in the present analysis were collected by self-administered questionnaires, and this method of data collection might lead to misclassifying the participants. These misclassifications would attenuated the original associations. Accordingly, it seems that the observed associations in the current study are much stronger in reality. The subjective or arbitrary deci- sions in factor analysis, such as the choice of somatic complaints to be included in the analysis, the number of factors to be extracted, and selecting the method of rotation should be considered while interpreting the results. ${ }^{63}$ Finally, since the SEPAHAN study's participants were 20 to 50 years of age working in health centers and not academic staff members of the IUMS, they may have a healthier lifestyle. Thus, generalization of the present findings to the general population in Iran, as well as to other cultural backgrounds, must be done with caution.

In conclusion, patients with CUD had a higher frequency of somatic complaints than control participants. Patients with CUD reported a greater frequency of severe fatigue and feeling low on energy. Also, we found that CUD and its symptoms are strongly associated with higher levels of all four somatic complaints profiles identified by factor analysis, especially the psychological, neuroskeletal, and pharyngeal-respiratory profiles. Our study's findings, in the context of an observational study, provide primary evidence about the usefulness of treating somatic complaints for targeting CUD's treatment. Also, these findings could be an indication of the key role of psychological factors on CUD and its symptoms, and may explain why it can be difficult to treat. However, further prospective particularly interventional investigations are required to confirm the impacts of treating of somatic complaints on CUD.

Acknowledgements: We are grateful to thank all staff of Isfahan University of Medical Sciences (MUI) who kindly participated in our study, and the staff of the Public Relations Unit, and other authorities of IUMS for their excellent cooperation.

Financial support: SEPAHAN was financially supported by a grant from the Vice Chancellery for Research and Technology, IUMS (Project No. 189069, 189082, and 189086).

\section{Conflicts of interest: None.}

Author contributions: Peyman Adibi, Awat Feizi, Ammar Hassanzadeh Keshteli, and Hamid Afshar contributed to the conception and design of the study, collection and assembly of the data; and Awat Feizi and Zahra Heidari contributed to the statistical analysis plan for the study, the analysis and interpretation of the data, and the drafting of the manuscript. All authors read and approved the final manuscript. 


\section{References}

1. Tack J, Bisschops R, Sarnelli G. Pathophysiology and treatment of functional dyspepsia. Gastroenterology 2004;127:1239-1255.

2. Tack J, Talley NJ, Camilleri M, et al. Functional gastroduodenal disorders. Gastroenterology 2006;130:1466-1479.

3. Brun R, Kuo B. Functional dyspepsia. Therap Adv Gastroenterol 2010;3:145-164

4. Goswami BD, Phukan C. Clinical features of functional dyspepsia. J Assoc Physicians India 2012;60:21-22.

5. Parkman HP, Camilleri M, Farrugia G, et al. Gastroparesis and functional dyspepsia: excerpts from the AGA/ANMS meeting. Neurogastroenterol Motil 2010;22:113-133.

6. Puri AS, Garg V. Differential diagnosis in functional dyspepsia. J Assoc Physicians 2012;60(suppl):23-24.

7. Talley NJ, Stanghellini V, Heading RC, Koch KL, Malagelada JR, Tytgat GN. Functional gastroduodenal disorders. Gut 1999;45(suppl 2):II37-II42.

8. El-Serag HB, Talley NJ. Health-related quality of life in functional dyspepsia. Aliment Pharmacol Ther 2003;18:387-393.

9. Koloski NA, Talley NJ, Huskic SS, Boyce PM. Predictors of conventional and alternative health care seeking for irritable bowel syndrome and functional dyspepsia. Aliment Pharmacol Ther 2003;17:841-851.

10. Moayyedi P, Mason J. Clinical and economic consequences of dyspepsia in the community. Gut 2002;50(suppl 4):iv10-iv12.

11. Moghimi-Dehkordi B, Vahedi M, Khoshkrood Mansoori B, et al. Economic burden of gastro-oesophageal reflux disease and dyspepsia: a community-based study. Arab J Gastroenterol 2011;12:86-89.

12. Wahass S, Khalil MS, Al Qurain AA, Yasawy MI. The impact of functional dyspepsia on health-related quality of life in Saudi patients. Saudi J Gastroenterol 2006;12:123-129.

13. Piacentino D, Cantarini R, Alfonsi M, et al. Psychopathological features of irritable bowel syndrome patients with and without functional dyspepsia: a cross sectional study. BMC Gastroenterol 2011;11:94.

14. Ghoshal UC, Singh R, Chang FY, Hou X, Wong BC, Kachintorn U. Epidemiology of uninvestigated and functional dyspepsia in Asia: facts and fiction. J Neurogastroenterol Motil 2011;17:235-244.

15. Zagari RM, Law GR, Fuccio L, et al. Epidemiology of functional dyspepsia and subgroups in the Italian general population: an endoscopic study. Gastroenterology 2010;138:1302-1311.

16. Sorouri M, Pourhoseingholi MA, Vahedi M, et al. Functional bowel disorders in Iranian population using Rome III criteria. Saudi J Gastroenterol 2010;16:154-160.

17. Clauwaert N, Jones MP, Holvoet L, et al. Associations between gastric sensorimotor function, depression, somatization, and symptom-based subgroups in functional gastroduodenal disorders: are all symptoms equal? Neurogastroenterol Motil 2012;24:1088-e565.

18. Gracie DJ, Bercik P, Morgan DG, et al. No increase in prevalence of somatization in functional vs organic dyspepsia: a cross-sectional survey. Neurogastroenterol Motil 2015;27:1024-1031.

19. Barry S, Dinan TG. Functional dyspepsia: are psychosocial factors of relevance? World J Gastroenterol 2006;12:2701-2707.

20. Faramarzi M, Kheirkhah F, Shokri-Shirvani J, Mosavi S, Zarini S. Psychological factors in patients with peptic ulcerand functional dyspepsia. Caspian J Intern Med 2014;5:71-76.

21. Filipović BF, Randjelovic T, Ille T, et al. Anxiety, personality traits and quality of life in functional dyspepsia-suffering patients. Eur J Intern Med 2013;24:83-86.

22. Lee SP, Sung IK, Kim JH, Lee SY, Park HS, Shim CS. The effect of emotional stress and depression on the prevalence of digestive diseases. J Neurogastroenterol Motil 2015;21:273-282.

23. Van Oudenhove L, Vandenberghe J, Geeraerts B, et al. Determinants of symptoms in functional dyspepsia: gastric sensorimotor function, psychosocial factors or somatisation? Gut 2008;57:1666-1673.

24. Lipowski ZJ. Somatization: the concept and its clinical application. Am J Psychiatry 1988;145:1358-1368.

25. Wilhelmsen I. Somatization, sensitization, and functional dyspepsia. Scand J Psychol 2002;43:177-180.

26. Rief W, Hiller W. A new approach to the assessment of the treatment effects of somatoform disorders. Psychosomatics 2003;44:492-498.

27. Fischler B, Tack J, De Gucht V, et al. Heterogeneity of symptom pattern, psychosocial factors, and pathophysiological mechanisms in severe functional dyspepsia. Gastroenterology 2003;124:903-910.

28. Jones MP, Sharp LK, Crowell MD. Psychosocial correlates of symptoms in functional dyspepsia. Clin Gastroenterol Hepatol 2005;3:521-528.

29. Bennett EJ, Piesse C, Palmer K, Badcock CA, Tennant CC, Kellow JE. Functional gastrointestinal disorders: psychological, social, and somatic features. Gut 1998;42:414-420.

30. Hsu YC, Liou JM, Liao SC, et al. Psychopathology and personality trait in subgroups of functional dyspepsia based on Rome III criteria. Am J Gastroenterol 2009;104:2534-2542.

31. Adibi P, Keshteli AH, Esmaillzadeh A, et al. The study on the epidemiology of psychological, alimentary health and nutrition (SEPAHAN): overview of methodology. J Res Med Sci 2012;17.

32. Esmaillzadeh A, Keshteli AH, Feizi A, Zaribaf F, Feinle-Bisset C, Adibi P. Patterns of diet-related practices and prevalence of gastro-esophageal reflux disease. Neurogastroenterol Motil 2013;25:831-e638.

33. Lacourt T, Houtveen J, van Doornen L. "Functional somatic syndromes, one or many?": an answer by cluster analysis. J Psychosom Res 2013;74:6-11.

34. Spitzer RL, Kroenke K, Williams JB. Validation and utility of a selfreport version of PRIME-MD: the PHQ primary care study. Jama 1999;282:1737-1744.

35. Spitzer RL, Williams JB, Kroenke K, Hornyak R, McMurray J. Validity and utility of the PRIME-MD patient health questionnaire in assessment of 3000 obstetric-gynecologic patients: the PRIME-MD Patient Health Questionnaire Obstetrics-Gynecology Study. Am J Obstet Gynecol 2000;183:759-769.

36. de Ruiter C, Garssen B, Rijken H, Kraaimaat F. The hyperventilation syndrome in panic disorder, agoraphobia and generalized anxiety disorder. Behav Res Ther 1989;27:447-452.

37. van Ravesteijn $H$, Wittkampf K, Lucassen $P$, et al. Detecting somatoform disorders in primary care with the PHQ-15. Ann Fam Med 2009; 7:232- 
238.

38. Montazeri A, Vahdaninia M, Ebrahimi M, Jarvandi S. The Hospital Anxiety and Depression Scale (HADS): translation and validation study of the Iranian version. Health Qual Life Outcomes 2003;1:14.

39. Montazeri A, Harirchi AM, Shariati M, Garmaroudi G, Ebadi M, Fateh A. The 12-item General Health Questionnaire (GHQ-12): translation and validation study of the Iranian version. Health Qual Life Outcomes 2003; 1:66.

40. Shaw A, McMunn A, Field J. Scottish health survey 1998. Edinburgh: The Stationery Office 2000.

41. Roohafza H, Ramezani M, Sadeghi M, Shahnam M, Zolfagari B, Sarafzadegan N. Development and validation of the stressful life event questionnaire. Int J Public Health 2011;56:441-448.

42. Cuijpers $P$, van Straten A, Donker M. Personality traits of patients with mood and anxiety disorders. Psychiatry Res 2005;133:229-37.

43. National Health. The General Practice Physical Activity Questionnaire (GPPAQ) 2006. Available from URL: http://www.ncbi.nlm.nih.gov/ books/NBK5 1962 (accessed 8 August, 2016).

44. Haug TT, Svebak S, Wilhelmsen I, Berstad A, Ursin H. Psychological factors and somatic symptoms in functional dyspepsia. A comparison with duodenal ulcer and healthy controls. J Psychosom Res 1994;38:281-291.

45. Norton GR, Norton PJ, Asmundson GJ, Thompson LA, Larsen DK. Neurotic butterflies in my stomach: the role of anxiety, anxiety sensitivity and depression in functional gastrointestinal disorders. J Psychosom Res 1999;47:233-240.

46. Van Oudenhove L, Holvoet L, Vandenberghe J, Vos R, Tack J. Do we have an alternative for the Rome III gastroduodenal symptom-based subgroups in functional gastroduodenal disorders? A cluster analysis approach. Neurogastroenterol Motil 2011;23:730-738.

47. Nan J, Liu J, Mu J, et al. Brain-based correlations between psychological factors and functional dyspepsia. J Neurogastroenterol Motil 2015;21:103-110.

48. Vishnar A, Ghulam R, Mittal RK. Non ulcer dyspepsia and its correlation with life stress, anxiety and depression. Indian J Psychiatry 2000;42:88-93.

49. De la Roca-Chiapas JM, Solís-Ortiz S, Fajardo-Araujo M, Sosa M, Córdova-Fraga T, Rosa-Zarate A. Stress profile, coping style, anxiety, depression, and gastric emptying as predictors of functional dyspepsia: a case-control study. J Psychosom Res 2010;68:73-81.

50. Holtmann G, Kutscher SU, Haag S, et al. Clinical presentation and personality factors are predictors of the response to treatment in patients with functional dyspepsia: a randomized, double-blind placebo-controlled crossover study. Dig Dis Sci 2004;49:672-679.

51. Piessevaux H, De Winter B, Louis E, et al. Dyspeptic symptoms in the general population: a factor and cluster analysis of symptom groupings. Neurogastroenterol Motil 2009;21:378-388.

52. Keohane J, Quigley EM. Functional dyspepsia and nonerosive reflux disease: clinical interactions and their implications. MedGenMed 2007;9:31.

53. Ford AC, Talley NJ, Schoenfeld PS, Quigley EM, Moayyedi P. Efficacy of antidepressants and psychological therapies in irritable bowel syndrome: systematic review and meta-analysis. Gut 2009;58:367-378.

54. Corsetti M, Caenepeel P, Fischler B, Janssens J, Tack J. Impact of coexisting irritable bowel syndrome on symptoms and pathophysiological mechanisms in functional dyspepsia. Am J Gastroenterol 2004;99:11521159.

55. Jiang SM, Jia L, Lei XG, et al. Incidence and psychological-behavioral characteristics of refractory functional dyspepsia: a large, multicenter, prospective investigation from China. World J Gastroenterol 2015;21:1932-1937.

56. De Gucht V, Maes S. Explaining medically unexplained symptoms: toward a multidimensional, theory-based approach to somatization. J Psychosom Res 2006;60:349-352.

57. Kindt S, Van Oudenhove L, Mispelon L, Caenepeel P, Arts J, Tack J. Longitudinal and cross-sectional factors associated with long-term clinical course in functional dyspepsia: a 5-year follow-up study. Am J Gastroenterol 2011;106:340-348.

58. Van Oudenhove L, Vandenberghe J, Vos R, Holvoet L, Tack J. Factors associated with co-morbid irritable bowel syndrome and chronic fatigue-like symptoms in functional dyspepsia. Neurogastroenterol Motil 2011;23:524-e202.

59. Powell N, Huntley B, Beech T, Knight W. Upper gastrointestinal symptoms and asthma: a manifestation of allergy? Gut 2008;57:1026-1027.

60. Siegel PD, Katz J. Respiratory complications of gastroesophageal reflux disease. Prim Care 1996;23:433-441.

61. Kroenke K, Spitzer RL, Williams JB. The PHQ-15: validity of a new measure for evaluating the severity of somatic symptoms. Psychosom Med 2002;64:258-266.

62. De Gucht V, Fischler B. Somatization: a critical review of conceptual and methodological issues. Psychosomatics 2002;43:1-9.

63. Martínez ME, Marshall JR, Sechrest L. Invited commentary: factor analysis and the search for objectivity. Am J Epidemiol 1998;148:17-19. 